\title{
Prevalence, risk factors and global impact of musculoskeletal injuries among endoscopists: a nationwide European study
}

\section{(ㄷ)(우우}

\section{Authors}

Rui Morais ${ }^{1}$, Filipe Vilas-Boas ${ }^{1}$, Pedro Pereira ${ }^{1}$, Pedro Lopes ${ }^{2}$, Carolina Simões ${ }^{3}$, Eduardo Dantas ${ }^{4}$, Inês Cunha ${ }^{5}$, Joana Roseira $^{6}$, João Cortez-Pinto ${ }^{7}$, João Silva ${ }^{8}$, Jorge Lage ${ }^{9}$, Mafalda Caine ${ }^{10}$, Manuel Rocha ${ }^{11}$, Margarida Flor de Lima ${ }^{12}$, Maria Pia Costa Santos ${ }^{13}$, Mónica Garrido ${ }^{14}$, Paula Sousa ${ }^{15}$, Pedro Marcos ${ }^{16}$, Richard Azevedo ${ }^{17}$, Rui Castro ${ }^{18}$, Tiago Cúrdia Gonçalves ${ }^{19,20,21}$, Tiago Leal ${ }^{22}$, Vítor Magno-Pereira ${ }^{23}$, Rosa Ramalho ${ }^{1}$, Eduardo Rodrigues-Pinto ${ }^{1}$, Guilherme Macedo ${ }^{1}$

\section{Institutions}

1 Gastroenterology Department, Centro Hospitalar Universitário de São João, Porto, Portugal

2 Physical Medicine and Rehabilitation Department, Centro Hospitalar Universitário de São João, Porto, Portugal

3 Gastroenterology Department, Centro Hospitalar Lisboa Norte, Lisboa, Portugal

4 Gastroenterology Department, Centro Hospitalar de Setúbal, Setúbal, Portugal

5 Gastroenterology Department, Centro Hospitalar e Universitário de Coimbra Coimbra, Portugal

6 Gastroenterology Department, Centro Hospitalar Universitário Algarve, Faro, Portugal

7 Gastroenterology Department, IPO Lisboa, Lisboa, Portugal

8 Gastroenterology Department, Centro Hospitalar Vila Nova de Gaia/Espinho, Vila Nova de Gaia, Portugal

9 Gastroenterology Department, Centro Hospitalar de Trás-os-Montes e Alto Douro, Vila Real, Portugal

10 Gastroenterology Department, IPO Coimbra, Coimbra, Portugal

11 Gastroenterology Department, Centro Hospitalar Lisboa Central, Lisboa, Portugal

12 Gastroenterology Department, Hospital do Divino Espírito Santo de Ponta Delgada, Ponta Delgada, Portugal

13 Gastroenterology Department, Hospital Beatriz Ângelo, Loures, Portugal

14 Gastroenterology Department, Centro Hospitalar Porto, Porto, Portugal

15 Gastroenterology Department, Centro Hospitalar Tondela-Viseu, Tondedla-Visue, Portugal

16 Gastroenterology Department, Centro Hospitalar de Leiria, Leiria, Portugal

17 Gastroenterology Department, Centro Hospitalar de Castelo-Branco, Castelo-Branco, Portugal

18 Gastroenterology Department, IPO Porto, Porto, Portugal
19 Gastroenterology Department, Hospital Senhora da Oliveira, Guimarães Braga, Portugal

20 Life and Health Sciences Research Institute, School of Medicine, University of Minho, Braga/Guimarães, Braga, Portugal

21 ICVS/3B's, PT Government Associate Laboratory, Braga/Guimarães, Braga, Portugal

22 Gastroenterology Department, Hospital de Braga, Braga, Portugal

23 Gastroenterology Department, Hospital Central do Funchal, Madeira, Portugal

submitted 9.8.2019

accepted after revision $\quad 15.10 .2019$

Bibliography

DOI https://doi.org/10.1055/a-1038-4343 |

Endoscopy International Open 2020; 08: E470-E480

(c) Georg Thieme Verlag KG Stuttgart · New York

elSSN 2196-9736

\section{Corresponding author}

Rui Morais, MD, Gastroenterology Department, Centro Hospitalar São João, Porto. Al. Prof. Hernâni Monteiro, 4200 - 319 Porto, Portugal

Fax: +351225513601

ruimorais20@gmail.com

$\circledast$ Supplementary material

Online content viewable at:

https://doi.org/10.1055/a-1038-4343

\section{ABSTRACT}

Background and study aims Previous studies have suggested a high prevalence of musculoskeletal injuries (MI) in endoscopists. Little evidence has come from European countries. Our main aim was to evaluate the prevalence, type, and impact of $\mathrm{Ml}$ among Portuguese endoscopists. 
We also sought to identify risk factors for the development, severity and number of endoscopy-related MI.

Material and methods A 48-question electronic survey was developed by a multidisciplinary group. The electronic survey was sent to all members of Portuguese Society of Gastroenterology $(n=705)$ during May 2019. Study data were collected and managed using REDCap electronic data capture tools hosted at SPG - CEREGA.

Results The survey was completed by 171 endoscopists (response rate of $24.3 \%$ ), $55.0 \%$ female with a median age of 36 years (range 26-78). The prevalence of at least one MI related to endoscopy was $69.6 \%(n=119)$, the most frequent being neck pain (30.4\%) and thumb pain $(29.2 \%)$.
The median time for MI development was 6 years (range 2 months-30 years). Severe pain was reported by $19.3 \%$. Change in endoscopic technique was undertaken by $61.3 \%$ and reduction in endoscopic caseload was undertaken by $22.7 \%$. Missing work was reported by $10.1 \%$, with the median time off from work being 30 days (range 1-90). Female gender and $\geq 15$ years in practice were independently associated with MI and severe pain. Years in practice, weeklytime performing endoscopy, and gender were significant predictors of the number of MI.

Conclusions Prevalence of MI was significant among Portuguese endoscopists and had a relevant impact on regular and professional activities.

\section{Introduction}

Self-reported musculoskeletal pain has been shown to affect up to $20 \%$ of adults in the general population and is a significant cause of disability that affects social functioning, mental health and quality of life (QoL) [1].

Occupational musculoskeletal injuries (MI) are an increasing problem among healthcare professionals [2-4]. Approximately $10 \%$ of workers in the European Union are healthcare employees, with this sector having the second-highest incidence rate of work-related MI, after construction [5]. A recent retrospective observational study based on the analysis of 1621 cases of work-related accidents of employees of Centro Hospitalar Universitário de São João (Porto, Portugal) from January 2011 to December 2014 identified a total of 824 cases of MI, which corresponded to a total of 22159 lost workdays [6].

Gastrointestinal endoscopy has assumed an exponential role in the diagnostic and therapeutic approach of conditions, with an increasing number of procedures performed worldwide [79]. The ever-growing performance of longer, more complex and technically challenging procedures such as endoscopic retrograde cholangiopancreatography (ERCP), endoscopic ultrasonography (EUS), endoscopic submucosal dissection (ESD) or natural orifice transluminal endoscopic surgery (NOTES) may predispose endoscopists to higher rates of overuse injuries than previously reported $[10,11]$.

Furthermore, the increasing scope of cancer screening programs will inevitably lead to an increase in demand for endoscopic procedures $[12,13]$.

Gastrointestinal endoscopy requires the physician to perform repetitive motions in challenging positions. Several studies and guidelines have been carried out on patient safety and quality of gastrointestinal endoscopy [8, 14]. However, less data are available on the safety and welfare of the endoscopists themselves, with little reported information about short-term and long-term disability from endoscopy-related MI [15]. Some studies have suggested a high prevalence of MI in endoscopists, including among fellows in training [16-23]. A systematic review estimated that $39 \%$ to $89 \%$ of endoscopists developed work-related MI [24]. A recent study among active members of the American Society for Gastrointestinal Endoscopy (ASGE) reported that $53 \%$ of endoscopists had a MI related to endoscopy and identified higher procedure volume, number of weekly hours performing endoscopy, and number of years performing endoscopy as factors associated with a higher rate of MI [10]. Nevertheless, ergonomic factors like bad posture during the procedure [18] or poor ergonomic design of facilities [16] have also been related to development of MI.

Despite previous American [10,17, 19, 22-23], Korean [18], and Japanese [20] reports on this topic, little evidence has come from European countries. Therefore, our main aim was to evaluate prevalence, type, and impact of $\mathrm{Ml}$ in regular and labor activity among Portuguese endoscopists. We also sought to identify risk factors for development, severity, and number of endoscopy-related MI.

\section{Material and methods}

We conducted an electronic survey directed to all endoscopists who are members of the Portuguese Society of Gastroenterology (SPG). Members who have ever performed endoscopy were eligible to participate. At least 6 months of endoscopy practice was required for participation. Informed consent was implied by the response to the survey. No financial compensation was given for participation. Ethical approval was obtained from the Ethics Committee for Health of the Centro Hospitalar Universitário de São João (Porto, Portugal).

\section{Survey instrument}

A 39-question, self-administered, electronic survey was developed by a group of gastroenterologists from 21 Portuguese centers and a physiatrist (Supplementary Table 1). Survey items were generated based on a review of the literature and multidisciplinary discussion. The survey was initially distributed, tested, and optimized among endoscopists of the Gastroenterology Department of Centro Hospitalar Universitário de São João to evaluate the content, clarity of the questions, and time necessary to complete the survey. The final survey evaluated endoscopist characteristics, workload parameters, type, treatment, and impact of MI (including the severity of worst 
pain ever felt assessed by the Numerical Rating Scale [NRS]). Severe pain was defined as NRS $\geq 8$ [25]. The survey required approximately 5 minutes to be filled out.

\section{Survey administration}

The electronic survey was sent to all members of SPG who met inclusion criteria $(n=705)$, during May 2019. Subjects were contacted via email to participate. The initial email included a cover letter explaining the study and a link to the survey. A reminder email was sent 2 weeks after the initial invitation. One month after the first email, the survey was terminated. All the answers remained anonymous. Study data were collected and managed using REDCap electronic data capture tools hosted at SPG - CEREGA.

\section{Statistical analysis}

All data were arranged, processed, and analyzed with SPSS v.24.0 data. For analysis of the characteristics of the endoscopists and endoscopy-related data, responders were classified into two main groups (those with or without MI). For analysis of prevalence, type, treatment and impact of MI, consultants and fellows were analyzed separately. Categorical variables were described through absolute and relative frequencies and continuous variables were described through mean, standard deviation, median, minimum and maximum. Hypotheses were tested using Fisher's exact test and Pearson chi-square test for categorical variables, independent sample $t$-test and one-way ANOVA for continuous variables with normal distribution and Mann-Whitney test and Kruskal-Wallis test for continuous variables with non-normal distribution.

Binary logistic regression was used to identify factors associated with $\mathrm{Ml}$ and factors associated with severe pain. Variables having $P<0.150$ on univariate analysis were incorporated into a stepwise multivariate regression model to confirm its independent association with the expected outcome. A stepwise multiple linear regression was performed to identify the variables associated with the number of MI. All significance levels were set at $P<0.05$.

\section{Results}

A total of 705 endoscopists (624 consultants and 81 fellows) were invited to participate. The survey was completed by 171 individuals (24.3\%). The response rate was higher among fellows compared to consultants (46.9\% vs. $21.3 \%$ ).

\section{Endoscopist characteristic}

Characteristics of endoscopists are summarized in $>$ Table 1. Fifty-five percent were female, with a median age of 36 years (range 26-78). Most of the respondents were currently performing endoscopy (97.7\%). Fifty-two percent worked in academic centers (AC), $41.5 \%$ in community centers (CC) and $63.7 \%$ in private practice (PP), with $56.1 \%$ having more than one work-setting simultaneously.

\section{Endoscopy-related data}

A detailed description of endoscopic procedure-related data and endoscopy workload parameters can be seen in $>$ Table 2 and Table 3, respectively. Median time of endoscopic practice was 9 years (range $0.5-45.0$ ). The majority performed esophagogastroduodenoscopy (EGD) and colonoscopy, 21.6\% ERCP and $21.6 \%$ EUS. Over $90 \%$ performed therapeutic endoscopic procedures.

Median weekly-working time was 50 hours (range 5-75). Median weekly-time performing endoscopy was 25 hours (range 3-52), with a higher proportion of time spent performing EGD and colonoscopy (median $30 \%$ and $60 \%$, respectively).

Most endoscopists took breaks (83\%) during endoscopic practice. The main usual environmental modification made during endoscopy was the use of a height-adjustable table (92.4\%).

\section{Prevalence and type of musculoskeletal injuries}

One hundred and twenty-nine respondents (75.4\%) reported having experienced $\mathrm{Ml}$ since the start of endoscopic practice. Prevalence of at least one $\mathrm{MI}$ related to endoscopy was $69.6 \%$ $(n=119)$ ( $\triangleright$ Table 4). Median time for MI development was 6 years (range 2 months-30 years). Median NRS value of the most painful area was 5 (range 1-10). Severe pain was reported by $19.3 \%$. Although there was no significant difference between prevalence of $\mathrm{MI}$ in consultants and fellows $(70.7 \%$ vs. $65.8 \%, P=0.555)$, consultants had a significantly higher number of MI (median 3 vs. 2, $P=0.007$ ). Except for the first year in practice, fellows were similarly distributed across the 4 years of their fellowship (first year: $n=4[10.5 \%]$; second year: $n=12$ [31.6\%]; third year: $n=9$ [23.7\%]; fourth year: $n=13$ [34.2\%]). Despite no available data on three fellows, in $50 \%(11 / 22) \mathrm{MI}$ occurred during the first year of fellowship, $27.3 \%$ in the second year, $18.2 \%$ in the thirrd year and $4.5 \%$ in the last year. The prevalence of MI did not differ significantly between each year of fellow in training [first year: $n=3 / 4(75 \%)$; second year: $n=6 / 12(50 \%)$; third year: $n=6 / 9(66.7 \%)$; fourth year: $n=10 /$ 13 (76.9\%); $P=0.532]$.

The most frequently reported types of $\mathrm{Ml}$ were neck pain (30.4\%) and thumb pain (29.2\%). Wrist pain and hand numbness were significantly more prevalent among consultants than fellows, with no significant differences regarding the remaining $\mathrm{MI}$.

\section{Impact of MI, practice modifications, and treatment}

A complete overview of the impact of MI, practice modifications and treatment is described in $\mathbf{T}$ Table 5. Almost half the respondents with $\mathrm{MI}(45.4 \%)$ reported symptoms during both work and daily activities, while $20.2 \%$ were only symptomatic during endoscopy. In $22.7 \%$, symptoms were present all the time. Change of endoscopic technique was undertaken by $61.3 \%$, with $7.0 \%$ reported sitting while performing endoscopy. The most commonly undertaken practice modification was using of adjustable bed (49.6\%), although $26.9 \%$ reported not undertaking any modification. Reduction of endoscopic caseload was performed in $22.7 \%$ of the respondents, with a signif- 
- Table 1 Characteristics of endoscopists.

\begin{tabular}{|c|c|c|c|c|}
\hline & $\begin{array}{l}\text { Total [n (\%)] } \\
n=171\end{array}$ & $\begin{array}{l}\text { No endoscopy-related } \\
\text { MI }[n(\%)] \\
n=52\end{array}$ & $\begin{array}{l}\text { Endoscopy-related } \\
\text { MI [n (\%)] } \\
n=119\end{array}$ & $P$ value \\
\hline Gender & & & & 0.068 \\
\hline - Female & $94(55.0 \%)$ & $23(44.2 \%)$ & $71(59.7 \%)$ & \\
\hline - Male & $77(45.0 \%)$ & $29(55.8 \%)$ & $48(40.3 \%)$ & \\
\hline Age, median (range), years & $36(26-78)$ & $34(26-76)$ & $37(27-78)$ & 0.076 \\
\hline Dominant hand & & & & 0.251 \\
\hline - Right & 157 (91.8\%) & $45(86.5 \%)$ & $112(94.1 \%)$ & \\
\hline - Left & $10(5.9 \%)$ & $5(9.6 \%)$ & $5(4.2 \%)$ & \\
\hline - Ambidexterity & $4(2.3 \%)$ & $2(3.9 \%)$ & $2(1.7 \%)$ & \\
\hline Glove size & & & & 0.734 \\
\hline - Small & $54(31.6 \%)$ & $15(28.9 \%)$ & $39(32.8 \%)$ & \\
\hline - Medium & $79(46.2 \%)$ & $23(44.2 \%)$ & $56(47.1 \%)$ & \\
\hline - Large & $34(19.9 \%)$ & $13(25.0 \%)$ & $21(17.6 \%)$ & \\
\hline - Extra-Large & $4(2.3 \%)$ & $1(1.9 \%)$ & $3(2.5 \%)$ & \\
\hline Physical activity level ${ }^{1}$ & & & & 0.725 \\
\hline - Vigorous & $5(2.9 \%)$ & $1(1.9 \%)$ & $4(3.4 \%)$ & \\
\hline - Moderate & $37(21.6 \%)$ & $12(23.1 \%)$ & $25(21.0 \%)$ & \\
\hline - Light & $81(47.4 \%)$ & $27(51.9 \%)$ & $54(45.4 \%)$ & \\
\hline - Sedentary & $48(28.1 \%)$ & $12(23.1 \%)$ & $36(30.2 \%)$ & \\
\hline Height, median (range), cm & $170(150-190)$ & $172(156-190)$ & $169(150-186)$ & 0.020 \\
\hline Weight, median (range), kg & $65(47-92)$ & $68.5(50-92)$ & $64(47-89)$ & 0.138 \\
\hline BMI, median (range), $\mathrm{kg} / \mathrm{m}^{2}$ & $22.6(17.6-31.6)$ & $22.3(18.1-31.5)$ & $22.7(17.6-31.6)$ & 0.817 \\
\hline Endoscopist's experience & & & & 0.564 \\
\hline - Fellow & $38(22.2 \%)$ & $13(25.0 \%)$ & $25(21.0 \%)$ & \\
\hline - Consultant & $133(77.8 \%)$ & $39(75.0 \%)$ & $94(79.0 \%)$ & \\
\hline Practice setting ${ }^{2}$ & & & & 0.491 \\
\hline - AC (only) & $29(17.0 \%)$ & $10(19.2 \%)$ & $19(16.0 \%)$ & \\
\hline - CC (only) & $32(18.7 \%)$ & $9(17.3 \%)$ & $23(19.3 \%)$ & \\
\hline - PP (only) & $14(8.2 \%)$ & $5(9.6 \%)$ & $9(7.6 \%)$ & \\
\hline - $A C+P P$ & $57(33.3 \%)$ & $19(36.6 \%)$ & $38(31.9 \%)$ & \\
\hline - $C C+P P$ & $36(21.0 \%)$ & $7(13.5 \%)$ & $29(24.4 \%)$ & \\
\hline - $A C+C C$ & $1(0.6 \%)$ & $1(1.9 \%)$ & $0(0.0 \%)$ & \\
\hline - $A C+C C+P P$ & $2(1.2 \%)$ & $1(1.9 \%)$ & $1(0.8 \%)$ & \\
\hline
\end{tabular}


- Table 2 Endoscopic procedure-related data.

\begin{tabular}{|c|c|c|c|c|}
\hline & $\begin{array}{l}\text { Total [n (\%)] } \\
n=171\end{array}$ & $\begin{array}{l}\text { No endoscopy-related MI } \\
\text { [n (\%)] } \\
n=52\end{array}$ & $\begin{array}{l}\text { Endoscopy-related MI } \\
\text { [n (\%)] } \\
n=119\end{array}$ & $P$ value \\
\hline Years in practice, median (range) & $9(0.5-45)$ & $6(0.5-44)$ & $9(0.5-45)$ & 0.027 \\
\hline \multicolumn{5}{|c|}{ Type of endoscopic procedures performed } \\
\hline EGD & & & & - \\
\hline - No & $0(0.0 \%)$ & $0(0.0 \%)$ & $0(0.0 \%)$ & \\
\hline - Yes & $171(100 \%)$ & $52(100.0 \%)$ & $119(100 \%)$ & \\
\hline Colonoscopy & & & & 0.347 \\
\hline - No & $2(1.2 \%)$ & $0(0.0 \%)$ & $2(1.7 \%)$ & \\
\hline - Yes & $169(98.8 \%)$ & $52(30.8 \%)$ & $117(98.3 \%)$ & \\
\hline ERCP & & & & 0.840 \\
\hline - No & $134(78.4 \%)$ & $40(76.9 \%)$ & $94(79.0 \%)$ & \\
\hline - Yes & $37(21.6 \%)$ & $12(23.1 \%)$ & $25(21.0 \%)$ & \\
\hline Diagnostic EUS & & & & 0.690 \\
\hline - No & $134(78.4 \%)$ & $42(80.8 \%)$ & $92(77.3 \%)$ & \\
\hline - Yes & $37(21.6 \%)$ & $10(19.2 \%)$ & $27(22.7 \%)$ & \\
\hline Therapeutic EUS & & & & 0.876 \\
\hline - No & $157(91.8 \%)$ & $48(92.3 \%)$ & $109(91.6 \%)$ & \\
\hline - Yes & $14(8.2 \%)$ & $4(7.7 \%)$ & $10(8.4 \%)$ & \\
\hline BAE & & & & 0.278 \\
\hline - No & $154(90.1 \%)$ & $49(94.2 \%)$ & $105(88.2 \%)$ & \\
\hline - Yes & $17(9.9 \%)$ & $3(5.8 \%)$ & $14(11.8 \%)$ & \\
\hline \multicolumn{5}{|l|}{ Therapeutic endoscopy } \\
\hline Therapeutic endoscopy & & & & 0.517 \\
\hline - No & $16(9.4 \%)$ & $6(11.5 \%)$ & $10(8.4 \%)$ & \\
\hline - Yes & $155(90.6 \%)$ & $46(88.5 \%)$ & $109(91.6 \%)$ & \\
\hline EMR & & & & 0.254 \\
\hline - No & $27(15.8 \%)$ & $11(21.2 \%)$ & $16(13.4 \%)$ & \\
\hline - Yes & $144(84.2 \%)$ & $41(78.8 \%)$ & $103(86.6 \%)$ & \\
\hline ESD & & & & 0.652 \\
\hline - No & $157(91.8 \%)$ & $47(90.4 \%)$ & $110(92.4 \%)$ & \\
\hline - Yes & $14(8.2 \%)$ & $5(9.6 \%)$ & $9(7.6 \%)$ & \\
\hline \multicolumn{5}{|l|}{ Endoscopic stenting } \\
\hline - No & $100(58.5 \%)$ & $31(59.6 \%)$ & $69(58.0 \%)$ & 0.842 \\
\hline - Yes & $71(41.5 \%)$ & $21(40.4 \%)$ & $50(42.0 \%)$ & \\
\hline Endoscopic dilation & & & & 0.405 \\
\hline - No & $79(46.2 \%)$ & $27(51.9 \%)$ & $52(43.7 \%)$ & \\
\hline - Yes & $92(53.8 \%)$ & $25(48.1 \%)$ & $67(56.3 \%)$ & \\
\hline
\end{tabular}


Table 2 (Continuation)

\begin{tabular}{|l|l|l|l|}
\hline & $\begin{array}{l}\text { Total [n (\%)] } \\
\mathbf{n = 1 7 1}\end{array}$ & $\begin{array}{l}\text { No endoscopy-related MI } \\
{[\mathbf{n}(\%)]} \\
\mathbf{n = 5 2}\end{array}$ & $\begin{array}{l}\text { Endoscopy-related MI } \\
{[\mathbf{n}(\%)]} \\
\mathbf{n}=\mathbf{1 1 9}\end{array}$ \\
\hline PEG & & & \\
\hline - No & $59(34.5 \%)$ & $21(40.4 \%)$ & $38(31.9 \%)$ \\
\hline - Yes & $112(65.5 \%)$ & $31(59.6 \%)$ & $81(68.1 \%)$ \\
\hline
\end{tabular}

MI, musculoskeletal injuries; EGD, esophagogastroduodenoscopy; ERCP, endoscopic retrograde cholangiopancreatography; EUS, endoscopic ultrasonography; BAE, Balloon-assisted enteroscopy; EMR, endoscopic mucosal resection; ESD, endoscopic submucosal dissection; PEG, percutaneous endoscopic gastrostomy

- Table 3 Endoscopy workload parameters.

\begin{tabular}{|c|c|c|c|c|}
\hline & $\begin{array}{l}\text { Total } \\
n=171\end{array}$ & $\begin{array}{l}\text { No endoscopy-related MI } \\
\text { [n (\%)] } \\
\mathrm{n}=52\end{array}$ & $\begin{array}{l}\text { Endoscopy-related MI } \\
{[\mathrm{n}(\%)]} \\
\mathrm{n}=119\end{array}$ & $P$ value \\
\hline Weekly-working time, median (range), hours & $50(5-75)$ & $52.5(15-75)$ & $50(5-70)$ & 0.171 \\
\hline $\begin{array}{l}\text { Weekly-time performing endoscopy, median } \\
\text { (range), hours }\end{array}$ & $25(3-52)$ & $25(10-50)$ & $25(3-52)$ & 0.361 \\
\hline $\begin{array}{l}\text { Weekly proportion of time Performing endoscopy, } \\
\text { median (range), \% }\end{array}$ & $50(15-100 \%)$ & $50(23-100 \%)$ & $50(15-95 \%)$ & 0.690 \\
\hline \multicolumn{5}{|c|}{ Proportion of time performing procedures, median (range), \% } \\
\hline - EGD & $30(5-100 \%)$ & $40(10-90 \%)$ & $30(5-100 \%)$ & 0.020 \\
\hline - Colonoscopy & $60(0-90 \%)$ & $50(10-90 \%)$ & $60(0-90 \%)$ & 0.030 \\
\hline - ERCP & $0(0-40 \%)$ & $0(0-40 \%)$ & $0(0-40 \%)$ & 0.644 \\
\hline - Diagnostic EUS & $0(0-40 \%)$ & $0(0-40 \%)$ & $0(0-32 \%)$ & 0.743 \\
\hline - Therapeutic EUS & $0(0-20 \%)$ & $0(0-8 \%)$ & $0(0-20 \%)$ & 0.952 \\
\hline - BAE & $0(0-20 \%)$ & $0(0-5 \%)$ & $0(0-20 \%)$ & 0.127 \\
\hline $\begin{array}{l}\text { Proportion of procedures under general } \\
\text { anesthesia median (range), \% }\end{array}$ & $60(0-100 \%)$ & $60(0-100 \%)$ & $63(0-100 \%)$ & 0.807 \\
\hline Breaks during endoscopy' & & & & 0.256 \\
\hline - Never & $29(17.0 \%)$ & $12(23.1 \%)$ & $17(14.3 \%)$ & \\
\hline - Occasionally & $126(73.7 \%)$ & $34(65.4 \%)$ & $92(77.3 \%)$ & \\
\hline - Frequently & $16(9.4 \%)$ & $6(11.5 \%)$ & $10(8.4 \%)$ & \\
\hline \multicolumn{5}{|l|}{ Environmental modifications during endoscopy ${ }^{2}$} \\
\hline - Height-adjustable table & $158(92.4 \%)$ & $48(92.3 \%)$ & $110(92.4 \%)$ & 0.977 \\
\hline - Monitor at eye level & $122(71.3 \%)$ & $40(76.9 \%)$ & $82(68.9 \%)$ & 0.359 \\
\hline - No helping to move patients & $51(29.8 \%)$ & $15(28.8 \%)$ & $36(30.3 \%)$ & 0.853 \\
\hline - Sitting & $12(7.0 \%)$ & $3(5.8 \%)$ & $9(7.6 \%)$ & 0.673 \\
\hline - None & $6(3.5 \%)$ & $1(1.9 \%)$ & $5(4.2 \%)$ & 0.456 \\
\hline $\begin{array}{l}\text { MI, musculoskeletal injuries; EGD, esophagogastroduodenos } \\
\text { Balloon-assisted enteroscopy } \\
{ }^{1} \text { Occasionally: } 1-2 \text { times per shift; Frequently: } 3-4 \text { times pe } \\
{ }^{2} \text { More than one may be present per endoscopist }\end{array}$ & \multicolumn{3}{|c|}{$\begin{array}{l}\text { MI, musculoskeletal injuries; EGD, esophagogastroduodenoscopy; ERCP, endoscopic retrograde cholangiopancreatography, EUs: endoscopic ultrasonography; BAE, } \\
\text { Balloon-assisted enteroscopy }\end{array}$} & phy; BAE, \\
\hline
\end{tabular}


- Table 4 Prevalence, characteristics, and types of endoscopy-related MI.

\begin{tabular}{|c|c|c|c|c|}
\hline & $\begin{array}{l}\text { Total [n (\%)] } \\
n=171\end{array}$ & $\begin{array}{l}\text { Consultants } \\
n(\%)] n=133\end{array}$ & $\begin{array}{l}\text { Fellows [n (\%)] } \\
n=38\end{array}$ & $P$ value \\
\hline Endoscopy-associated MI & $119(69.6 \%)$ & $94(70.7 \%)$ & $25(65.8 \%)$ & 0.555 \\
\hline Number of MI, median (range) & $2(1-8)$ & $3(1-8)$ & $2(1-4)$ & 0.007 \\
\hline $\begin{array}{l}\text { Time to Ml after starting endoscopy } \\
\text { practice, median (range), years }\end{array}$ & 6 [0 (2 months)-30] & 9 [0 (2 months)-30] & 1 [0 (4 months)-3] & $<0.001$ \\
\hline $\begin{array}{l}\text { Maximum pain severity, median (range), } \\
\text { NRS }\end{array}$ & $5(1-10)$ & $5(1-10)$ & $5(2-9)$ & 0.120 \\
\hline \multicolumn{5}{|l|}{ Types of endoscopy-associated MI ${ }^{1}$} \\
\hline Thumb pain & $50(29.2 \%)$ & $41(30.8 \%)$ & $9(23.7 \%)$ & 0.427 \\
\hline Wrist pain & $46(26.9 \%)$ & $41(30.8 \%)$ & $5(13.2 \%)$ & 0.037 \\
\hline Hand pain & $25(14.6 \%)$ & $18(13.5 \%)$ & $7(18.4 \%)$ & 0.443 \\
\hline Shoulder pain & $47(27.5 \%)$ & $41(30.8 \%)$ & $6(15.8 \%)$ & 0.098 \\
\hline Elbow pain & $16(9.4 \%)$ & $15(11.3 \%)$ & $1(2.6 \%)$ & 0.107 \\
\hline Neck pain & $52(30.4 \%)$ & $39(29.3 \%)$ & $13(34.2 \%)$ & 0.555 \\
\hline Thoracic back pain & $26(15.2 \%)$ & $23(17.3 \%)$ & $3(7.9 \%)$ & 0.204 \\
\hline Lower back pain & $30(17.5 \%)$ & $27(20.3 \%)$ & $3(7.9 \%)$ & 0.092 \\
\hline Hand numbness & $21(12.3 \%)$ & $20(15.0 \%)$ & $1(2.6 \%)$ & 0.048 \\
\hline
\end{tabular}

icantly higher proportion in consultants than in fellows $(27.7 \%$ vs. $4.0 \%, P=0.014)$. Missing work was reported by $10.1 \%$, with the median time off from work being 30 days (range 1-90). In $33.6 \%$, MI led to a reduction in physical activity (PA) outside of work.

In $25.2 \%$, no targeted treatment was performed, especially in fellows $(56.0 \%$ vs. $17.0 \%$ in consultants, $P<0.001)$. The most common specific treatments performed included NSAIDs (57.1\%) and physiotherapy (30.3\%). Surgery was performed on two endoscopists ( $>$ Fig. 1 ).

One hundred and forty-five respondents (84.8\%) would be interested in having their work setting assessed ergonomically, while 128 (74.9\%) would like to receive preventive information regarding $\mathrm{MI}$.

\section{Predictive factors for MI}

A multivariable regression model was developed to predict factors associated with endoscopy-related MI (Supplementary Table 2). Female gender (odds ratio [OR] 2.443, $95 \%$ confidence interval $[\mathrm{Cl}] 1.166-5.121 ; P=0.018), \geq 15$ years in practice (OR 3.514; $95 \% \mathrm{Cl} 1.490-8.284 ; P=0.004$ ) and the proportion of time performing EGD (OR 0.974 , $95 \% \mathrm{Cl} 0.951-$ $0.997 ; P=0.026$ ) were independently associated with MI.

\section{Predictive factors of severe pain}

Sub-analysis of respondents with MI was performed to identify predictive factors of severe pain, independently of lesion location (Supplementary Table 3). Female gender (OR 3.598, 95\%
CI 1.162-11.137; $P=0.026$ ), moderate/vigorous PA level (OR $3.318,95 \% \mathrm{Cl} 1.056-10.424 ; P=0.040)$ and $\geq 15$ years in practice (OR 4.284, $95 \% \mathrm{Cl} 1.440-12.745 ; P=0.009$ ) were independently associated with severe pain.

\section{Predictive factors of the number of MI}

Simple and multiple linear regression was performed to identify the variables associated with the number of MI (Supplementary Table 4).

Backward linear regression was performed, with the age, gender, endoscopist's experience, years in practice, weeklytime performing endoscopy and practice settings forced in the model. A significant regression equation was found $(F(3,111)=$ $7.221, P<0.001)$, with an R2 of 0.163 . Respondent's predicted number of $\mathrm{Ml}$ was equal to $0.195+0.522$ (gender) +0.042 (years in practice) +0.041 (weekly-time performing endoscopy), where gender was coded as $1=$ Male, $2=$ Female, years in practice was measured in years and weekly-time performing endoscopy was measured in hours. The number of $\mathrm{Ml}$ increased 0.042 for each year of practice, 0.041 for each weekly-hour performing endoscopy and females had 0.522 more MI than males. Years in practice $(P<0.001)$, weekly-time performing endoscopy $(p=0.005)$ and gender $(P=0.049)$ were significant predictors of the number of MI. 
- Table 5 Ml characterization, impact, and treatment.

\begin{tabular}{|c|c|c|c|c|}
\hline & $\begin{array}{l}\text { Total }[n(\%)] \\
n=119\end{array}$ & $\begin{array}{l}\text { Consultants }[\mathrm{n}(\%)] \\
\mathrm{n}=94\end{array}$ & $\begin{array}{l}\text { Fellows }[\mathrm{n}(\%)] \\
\mathrm{n}=25\end{array}$ & $P$ value \\
\hline MI associated-pain timing & & & & 0.286 \\
\hline - Work, during endoscopy & $24(20.2 \%)$ & $16(17.0 \%)$ & $8(32.0 \%)$ & \\
\hline Work, all the time & $8(6.7 \%)$ & $5(5.3 \%)$ & $3(12.0 \%)$ & \\
\hline - Work + daily activities & $54(45.4 \%)$ & $46(49.0 \%)$ & $8(32.0 \%)$ & \\
\hline - Daily activities only & $3(2.5 \%)$ & $2(2.1 \%)$ & $1(4.0 \%)$ & \\
\hline - All the time, including at rest & $27(22.7 \%)$ & $22(23.4 \%)$ & $5(20.0 \%)$ & \\
\hline - No data & $3(2.5 \%)$ & $3(3.2 \%)$ & - & \\
\hline Changes in endoscopic technique & & & & 0.646 \\
\hline - Yes & $73(61.3 \%)$ & $59(62.8 \%)$ & $14(56.0 \%)$ & \\
\hline - Not possible to change technique & $17(14.3 \%)$ & $12(12.8 \%)$ & $5(20.0 \%)$ & \\
\hline - Change of technique not tried & $27(22.7 \%)$ & $21(22.3 \%)$ & $6(24.0 \%)$ & \\
\hline - No data & $2(1.7 \%)$ & $2(2.1 \%)$ & - & \\
\hline \multicolumn{5}{|l|}{ Practice modification ${ }^{1}$} \\
\hline - Less endoscopy & $27(22.7 \%)$ & $26(27.7 \%)$ & $1(4.0 \%)$ & 0.014 \\
\hline - Stretching exercises before endoscopy & $20(16.8 \%)$ & $16(17.0 \%)$ & $4(16.0 \%)$ & 0.903 \\
\hline - More breaks between procedures & $26(21.8 \%)$ & $23(24.5 \%)$ & $3(12.0 \%)$ & 0.276 \\
\hline - Adjustable bed & $59(49.6 \%)$ & $45(47.9 \%)$ & $14(56.0 \%)$ & 0.470 \\
\hline - Orthopedic shoes & $18(15.1 \%)$ & $15(16.0 \%)$ & $3(12.0 \%)$ & 0.624 \\
\hline - No modification & $32(26.9 \%)$ & $25(26.6 \%)$ & $7(28.0 \%)$ & 0.888 \\
\hline \multicolumn{5}{|l|}{ Treatment $^{1}$} \\
\hline " NSAIDs & $68(57.1 \%)$ & $61(64.9 \%)$ & $7(28.0 \%)$ & 0.001 \\
\hline - Paracetamol & $21(17.6 \%)$ & $17(18.1 \%)$ & $4(16.0 \%)$ & 0.808 \\
\hline - Corticosteroid injection & $15(12.6 \%)$ & $14(14.9 \%)$ & $1(4.0 \%)$ & 0.145 \\
\hline - Parenteral corticosteroid & $2(1.7 \%)$ & $2(2.1 \%)$ & $0(0.0 \%)$ & 0.462 \\
\hline - Other medications ${ }^{2}$ & $12(10.1 \%)$ & $10(10.6 \%)$ & $2(8.0 \%)$ & 0.697 \\
\hline - Physiotherapy & $36(30.3 \%)$ & $33(35.1 \%)$ & $3(12.0 \%)$ & 0.028 \\
\hline - Splinting & $12(10.1 \%)$ & $12(12.8 \%)$ & $0(0.0 \%)$ & 0.060 \\
\hline - Surgery & $2(1.7 \%)$ & $2(2.1 \%)$ & $0(0.0 \%)$ & 0.462 \\
\hline - Rest & $35(29.4 \%)$ & $30(31.9 \%)$ & $5(20.0 \%)$ & 0.326 \\
\hline - No treatment & $30(25.2 \%)$ & $16(17.0 \%)$ & $14(56.0 \%)$ & $<0.001$ \\
\hline Missing work & $12(10.1 \%)$ & $11(11.7 \%)$ & $1(4.0 \%)$ & 0.234 \\
\hline $\begin{array}{l}\text { Missing work (maximum consecutive days), median } \\
\text { (range) }\end{array}$ & $30(1-90)$ & $30(1-90)$ & - & - \\
\hline Missing work (total number of days), median (range) & $30(1-90)$ & $35(1-90)$ & - & - \\
\hline Reduction in physical activity outside of work ${ }^{3}$ & $40(33.6 \%)$ & $35(37.2 \%)$ & $5(20.0 \%$ & 0.152 \\
\hline \multicolumn{5}{|c|}{$\begin{array}{l}1 \text { More than one may be present per endoscopist } \\
{ }^{2} \text { Tramadol } n=2 \text {; tramadol + paracetamol } n=1 \text {; paracetamol + thiocolchicoside } n=1 \text {; paracetamol + codeine } n=1 \text {; diazepam } n=3 \text {; topical NSAIDs } n=2 \text {; cyclobenzapr- } \\
\text { ine chloridrate } n=1 \text {; oral corticosteroids } n=1\end{array}$} \\
\hline
\end{tabular}



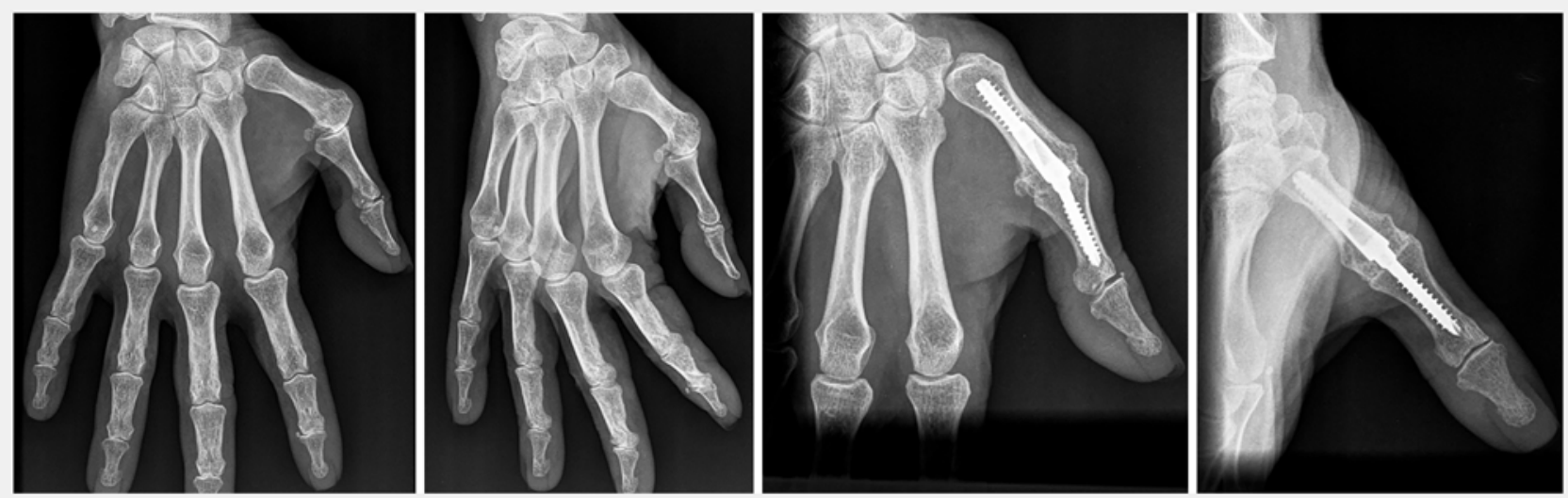

- Fig. 1 A female endoscopist with 20 years in practice reported progressive limitation of left hand metacarpophalangeal joint motion associated with pain, especially during colonoscopy performance. X-ray revealed subluxation of the metacarpophalangeal joint, with associated arthrosis. Multiple treatments were tried, including corticosteroid injections, topical and oral NSAIDs and use of splinting during procedures, without symptomatic improvement. The endoscopist underwent metacarpophalangeal joint arthrodesis, with excellent clinical and functional outcome.

\section{Discussion}

Endoscopists are at risk for MI, which can lead to loss of productivity and possibly career shortening [15]. Surprisingly, considering the negative impact of its occurrence, there is practically no data from European centers, with only one previous letter to the editor published by Geraghty et al reporting pain more than once a week during endoscopy in $57 \%$ of a group of 58 gastroenterologists surveyed in the northwest of England [26].

Cultural differences in the way work-related pain and injury are perceived were previously described, even within the same occupation [27]. On the other hand, differences in endoscopy practice are known, even among different European countries [7]. Except for the study by Ridtitid et al [10] that evaluated all active members of ASGE, quality issues in previous studies arose mainly from the fact that the survey responders were selfselected, being unclear if that led to response modulation [24]. Taking these into account, we performed a nationwide study on this topic. Prevalence of endoscopy-related MI was 69.6\%, significantly higher compared to most of the previous studies, including the survey among all ASGE members (prevalence of $53 \%$ ) [10] and the study among Japanese endoscopists (prevalence of $43 \%$ ) [20], but inferior to the study in Korean endoscopists $(89.1 \%)$ [18]. It is important to note that the workload among respondents in our study was significant, with a median of 25 hours per week spent performing endoscopy. More than half had more than one work-setting, often reconciling the work in an AC or CC with PP. Although no direct comparison can be made, this may be one of the possible explanations for the high prevalence of MI in our survey.

Despite the fact that most of the published data focused on longtime endoscopy practitioners, recent studies analyzed risk of MI among fellows. Prevalence of MI varied between $20 \%$ [23] to $47 \%$ [22]. In our study, the prevalence of MI among fellows was $65.8 \%$ with $77.3 \%$ of the cases occurring during the first two years of fellowship. One could argue that the endoscopic workload in Portuguese fellows is higher than in these studies, however considering the low number of procedures performed until MI development, specific unique factors unrelated to endoscopy workload and time in practice are likely associated with MI [23]. Ende et al analyzed force application during colonoscopy using a novel device and concluded that experts used higher average forward forces during insertion compared to all trainees and significantly less clockwise torque maneuver compared to novice trainees [28]. Differences in endoscopic technique can lead to development of specific MI. Some studies previously reported differences in location of MI between beginners and experienced endoscopists [18]. In our study, prevalence of hand numbness and wrist pain were significantly higher in consultants, while neck and hand pain were more frequent in fellows (although not reaching statistical significance). Interestingly, in response to $\mathrm{MI}$, reduction in endoscopic caseload and treatment were rarely undertaken by fellows compared to consultants. To some extent, this may reflect reluctance to acknowledge the occurrence of the lesion or seek help, with associated fear of seeing evolution as an endoscopist hindered or delayed.

Nevertheless, the repetitive and cumulative nature of the physical maneuvers involved in endoscopy can have long-term consequences, suggesting the importance of proper training in endoscopic technique as well as in ergonomics since fellowship [15]. In Portugal, there is no specific training in ergonomics. In the study by Villa $\mathrm{E}$ et al, prevalence of MI was significantly lower among fellows who received ergonomics training [22] suggesting that lack of integration of ergonomics in training can be one of the possible contributors to a higher prevalence of $\mathrm{Ml}$ among fellows in our study.

In previous studies, the most consistent risk factors associated with MI were endoscopic workload (measured by procedure volume or proportion of time spent performing endoscopy) and cumulative time spent performing endoscopy [24]. In our 
study, although we did not evaluate the total number of endoscopic procedures performed, we used the total weekly time performing endoscopy and the percentage of time performing endoscopy and specific endoscopic procedures as surrogate markers for the endoscopy workload. We identified female gender and $\geq 15$ years in practice as independent risk factors for MI, severe pain and a higher number of MI. Even though a recent study performed among ASGE fellows reported female gender as the only significant risk factor for MI [23], most of the prior studies have shown no gender differences in Ml risk, which could be limited by the low number of women included. In our study, more than half of the respondents were women, a number far higher than, for example, in the study by Ridtitid et al, which had $11.9 \%$ women respondents [10]. Higher risk of MI in women may be related to differences in hand size and grip strength. In a study performed among 227 fellows, $41.0 \%$ considered their hand too small for a standard endoscope, $78.2 \%$ considered that hand size affected endoscopy learning, and $34.2 \%$ would use smaller endoscopes if possible. Ninety-seven percent of the respondents with smaller hands were women [29]. A previous study reported women's grip strength to be $59 \%$ of men's and fingertip-pinch strength to be $71 \%$ to $73 \%$ of men's [30]. In our study, we identified female gender as a significant predictor not only for prevalence but also for severity and number of MI. In Portugal the female:male ratio in gastroenterologists increased from 0.5 among doctors aged 51 to 65 to 1.6 among those with aged 31 to 50 [31]. It is crucial to identify potentially modifiable factors (such as the ergonomics of the endoscopy suites or even the design of the endoscope) [15] that would allow for risk reduction of $\mathrm{MI}$ in female endoscopists.

Regarding the remaining predictors of $\mathrm{MI}$, a higher proportion of time performing EGD was identified as a protective factor. One can assume that endoscopists performing more EGD performed significantly fewer more technically demanding procedures, including colonoscopy, that have previously been associated with an increased risk of MI [32]. Moderate/vigorous PA was also a risk factor for severe pain. Nevertheless, a response bias cannot be excluded because respondents may have increased PA in response to $\mathrm{MI}$ development or, on the other hand, a higher level of PA could have led to exacerbation of Ml symptoms.

Although most studies focused on prevalence of endoscopic-related MI, few have evaluated its impact not only in work but also on regular activities. In our study, missing work was reported by $10.1 \%$ of respondents, with a median of 30 days off work. These results contrast with previous studies, in which few endoscopists reported missing work and when necessary, it usually lasted a few days $[17,19,26]$. On the other hand, $33.6 \%$ of respondents had to reduce PA outside work. These data highlight the possible negative impact of MI on regular daily-life activities. Additionally, MI can have a "domino effect" [19] and may affect not only the individual but also colleagues or even family relationships. Further studies are necessary to evaluate psychological morbidity and real impact on the QoL of these injuries.
In our study, $84.8 \%$ of respondents would have liked to have their work setting assessed ergonomically and $74.9 \%$ would have liked to receive preventive information regarding MI. The term "ergonomics" originally comes from the Greek words ergon (work) and nomos (natural laws) and relates to the study and optimization of the interactions between the worker, the equipment and the work environment. The importance of the design of the endoscopy suite for MI has previously been emphasized, with the ASGE guidelines on ergonomics recommending that the workplace should accommodate the fifth percentile female to the 95th percentile male [33]. On the other hand, some authors argue that endoscopists should be trained like athletes concerning ergonomics (the "endo-athlete") to further prevent endoscopy-related MI [34, 35]. Our results highlight the fact that despite lack of a formal training program in ergonomics, it is a matter of interest for endoscopists in our country. In a survey of 826 American Gastroenterology Association endoscopists, only $4.5 \%$ received ergonomics training during the fellowship [36]. ASGE guidelines may have been the starting point for a paradigm shift [32], with apparent beneficial effect already evident in the survey by Villa et al [22].

In our study, the number of participants was significant. Limitations of our study included a survey response rate of $24.3 \%$ which may subject the study to bias, making interpretation of results more challenging. Nevertheless, despite being modest, the response rate was superior compared to the other nationwide studies previously performed $[10,23]$. Other limitations need to be addressed, namely response and recall bias inherent in this type of study. Endoscopists with MI may have been more prone to participate in the study and overestimate the role of endoscopy in MI development. On the other hand, endoscopists working at an AC may have had a different attitude toward research, and may have been less likely than those in community settings or private practice to be non-respondents (whether having experienced MI or not). Regarding predictive factors for severe pain, the specific type of lesion associated with symptoms was not evaluated, thus limiting our conclusions. Finally, it was not possible to evaluate the role of more complex procedures such as ERCP, EUS or ESD in MI development due to the low number of endoscopists performing them procedures. One area of interest would be to study development of specific lesions after the beginning of the practice of particular techniques, as well as the timing of their occurrence.

\section{Conclusion}

In conclusion, this is the first nationwide European study that evaluated endoscopy-related MI. Prevalence of MI was significant among Portuguese endoscopists and had a relevant impact on regular and professional activities. An urgent effort by National and European societies is critical for development of a training program and implementation of guidelines in ergonomics that can contribute to reducing the burden of these injuries. 
Competing interests

The authors declare that they have no conflict of interest.

References

[1] Woolf AD, Pfleger B. Burden of major musculoskeletal conditions. Bull World Health Organ 2003; 81: 646-656

[2] Epstein S, Sparer EH, Tran BN et al. Prevalence of work-related musculoskeletal disorders among surgeons and interventionalists: a systematic review and meta-analysis. JAMA Surg 2018; 153: e174947

[3] Davis KG, Kotowski SE. Prevalence of musculoskeletal disorders for nurses in hospitals, long-term care facilities, and home health care: a comprehensive review. Hum Factors 2015; 57: 754-792

[4] Oranye NO, Bennett J. Prevalence of work-related musculoskeletal and non-musculoskeletal injuries in health care workers: the implications for work disability management. Ergonomics 2018; 61: 355366

[5] Factsheet 29 - Safety and health good practice online for the healthcare sector. Available from: https://osha.europa.eu/en/publications/ factsheet-29-safety-and-health-good-practice-line-healthcare-sector/view

[6] Amaro J, Magalhães J, Leite $M$ et al. Musculoskeletal injuries and absenteeism among healthcare professionals-ICD-10 characterization. PLoS One 2018; 13: e0207837

[7] Bisschops R, Wilmer A, Tack J. A survey on gastroenterology training in Europe. Gut 2002; 50: 724-729

[8] Rutter MD, Senore C, Bisschops R et al. The European Society of Gastrointestinal Endoscopy Quality Improvement Initiative: developing performance measures. United Eur Gastroenterol J 2016; 4: 30-41

[9] Peery AF, Crockett SD, Murphy CC et al. Burden and cost of gastrointestinal, liver, and pancreatic diseases in the United States: Update 2018. Gastroenterology 2019; 156: 254-272.e11

[10] Ridtitid W, Coté GA, Leung W et al. Prevalence and risk factors for musculoskeletal injuries related to endoscopy. Gastrointest Endosc 2015; 81: 294-302

[11] Nabi Z, Nageshwar Reddy D, Ramchandani M. Recent advances in third-space endoscopy. Gastroenterol Hepatol (N Y) 2018; 14: 224 232

[12] Areia M, Spaander MC, Kuipers EJ et al. Endoscopic screening for gastric cancer: a cost-utility analysis for countries with an intermediate gastric cancer risk. United European Gastroenterol J 2018; 6: 192-202

[13] Wolf AMD, Fontham ETH, Church TR et al. Colorectal cancer screening for average-risk adults: 2018 guideline update from the American Cancer Society. CA Cancer J Clin 2018; 68: 250-281

[14] Day LW, Cohen J et al. ASGE Endoscopy Unit Quality Indicator Taskforce. Quality indicators for gastrointestinal endoscopy units. VideoGIE 2017; 2: 119-140

[15] Harvin G. Review of musculoskeletal injuries and prevention in the endoscopy practitioner. J Clin Gastroenterol 2014; 48: 590-594

[16] O'Sullivan S, Bridge G, Ponich T. Musculoskeletal injuries among ERCP endoscopists in Canada. Can J Gastroenterol 2002; 16: 369-374

[17] Liberman AS, Shrier I, Gordon PH. Injuries sustained by colorectal surgeons performing colonoscopy. Surg Endosc 2005; 19: 16061609
[18] Byun YH, Lee JH, Park MK et al. Procedure-related musculoskeletal symptoms in gastrointestinal endoscopists in Korea. World J Gastroenterol 2008; 14: 4359-4364

[19] Hansel SL, Crowell MD, Pardi DS et al. Prevalence and impact of musculoskeletal injury among endoscopists: a controlled pilot study. J Clin Gastroenterol 2009; 43: 399-404

[20] Kuwabara T, Urabe Y, Hiyama T et al. Prevalence and impact of musculoskeletal pain in Japanese gastrointestinal endoscopists: a controlled study. World J Gastroenterol 2011; 17: 1488-1493

[21] Buschbacher R. Overuse syndromes among endoscopists. Endoscopy 1994; 26: 539-544

[22] Villa E, Attar B, Trick W et al. Endoscopy-related musculoskeletal injuries in gastroenterology fellows. Endosc Int Open 2019; 7: E808-E812

[23] Austin K, Schoenberger H, Sesto M et al. Musculoskeletal injuries are commonly reported among gastroenterology trainees: results of a national survey. Dig Dis Sci 2019; 64: 1439-1447

[24] Yung DE, Banfi T, Ciuti G et al. Musculoskeletal injuries in gastrointestinal endoscopists: a systematic review. Expert Rev Gastroenterol Hepatol 2017; 11: 939-947

[25] Boonstra AM, Stewart RE, Köke A] et al. Cut-off points for mild, moderate, and severe pain on the numeric rating scale for pain in patients with chronic musculoskeletal pain: variability and influence of sex and catastrophizing. Front Psychol 2016; 7: 1466

[26] Geraghty J, George R, Babbs C. A questionnaire study assessing overuse injuries in United Kingdom endoscopists and any effect from the introduction of the National Bowel Cancer Screening Program on these injuries. Gastrointest Endosc 2011; 73: 1069-1070

[27] Coggon D, Ntani G, Palmer KT et al. Disabling musculoskeletal pain in working populations: is it the job, the person, or the culture? Pain 2013; 154: 856-863

[28] Ende AR, De Groen P, Balmadrid BL et al. Objective Differences in Colonoscopy Technique Between Trainee and Expert Endoscopists Using the Colonoscopy Force Monitor. Dig Dis Sci 2018; 63: 46

[29] Cohen DL, Naik JR, Tamariz LJ et al. The perception of gastroenterology fellows towards the relationship between hand size and endoscopic training. Dig Dis Sci 2008; 53: 1902-1909

[30] Puh U. Age-related and sex-related differences in hand and pinch grip strength in adults. Int J Rehabil Res 2010; 33: 4-11

[31] Ordem dos Médicos. Portugal. National statistics by medical specialty. 2018: Available from: https://ordemdosmedicos.pt/wp-content/ uploads/2019/09/Distribui\%C3\%A7\%C3\%A3o-Especialidades_idadesexo_2018.pdf

[32] Shergill AK, Asundi KR, Barr A et al. Pinch force and forearm-muscle load during routine colonoscopy: a pilot study. Gastrointest Endosc 2009; 69: 142-146

[33] Pedrosa MC, Farraye FA, Shergill AK et al. Minimizing occupational hazards in endoscopy: personal protective equipment, radiation safety, and ergonomics. Gastrointest Endosc 2010; 72: 227-235

[34] Singla M, Kwok R, Deriban G et al. Training the endo-athlete: An update in ergonomics in endoscopy. Clin Gastro and Hepatol 2018; 16: 1003-1006

[35] Siau K, Anderson JT. Ergonomics in endoscopy: Should the endoscopist be considered and trained like an athlete? Endosc Int Open 2019; 7: E813-E815

[36] Edelman KM, Zheng J, Erdmann A et al. Endoscopy-related musculoskeletal injury in AGA gastroenterologists is common while training in ergonomics is rare. Gastroenterology 2017; 152: \$217 